\title{
Estudio del Efecto, Tamaño, Peso del Huevo sobre la Incubabilidad de broilers
}

RESPONSABLE: $M g r$. Cs. Daniel Gandarillas Espezua

\begin{abstract}
RESUMEN Setrabajaron con huevos procedentes de gallinas reproductoras de la linea Coob, con edades de 22 a 32 semanas, fueron categorizados como sigue: huevos pequeños con 41.09 - 50.97 grs., medianos: 50.98 - 57.39 grs. $y$ huevos grandes: 57.40 - 69.64 grs. Se consideraron tres tratamientos con tres replicaciones por tratamiento. Este trabajo de investigación probó la hipótesis de que el peso y tamaño del huevo influyen en la incubabilidad de pollos broilers. Los valores obtenidos para la pérdida de peso de las tres categorias (grande, mediano y pequeño): se registró un valor más alto en huevos medianos con relación a los otros; sin embargo, los huevos grandes registraron valores menores. No habiendo diferencias estadisticas significativas entre grupos. Referente al porcentaje de incubabilidad se encontraron valores entre 82.88 a 96.65 , siendo el mayor encontrado en huevos de tamaño mediano y menor en huevos de tamaño grande. Estos resultados demuestran que los huevos de tamaño mediano obtuvieron mejores resultados con relación a huevos de tamaño pequeño y grande.
\end{abstract}

\begin{abstract}
He worked with eggs from breeder hens line Cobb, aged 22 to 32 weeks, were categorized as follows: small eggs $41.09-50.97 \mathrm{gr}$., Medium: 50.98 - 57. 39 gr: And large eggs: 57.40 - 69.64 gr: It considered three treatments with three replicates for treatment. This research tested the hypothesis that weight and egg size affect the hatchability of chicken broillers. The values obtained for the weight loss of three categories (large, medium and small) recorded the highest value at medium eggs in relation to others, however large eggs showed lower values. There being no statistically significant differences between groups. Concerning the percentage of hatchability values were found between 82.88 to 96.65 , the largest being found in eggs of medium size and smaller in egg size. These results show that medium-sized eggs performed better compared to eggs of both large and small
\end{abstract}

INTRODUCCIÓN. En la producción de huevos de tipo comercial se requieren huevos de mayor tamaño y peso, y el precio de estos son mayores con relación a los de menor tamaño y peso. Para la producción de pollos broilers (parrilleros) se requieren huevos de tamaño medio y alto, con pesos arriba de 50 gramos.

En el ciclo de producción de la gallina ponedora, la gallina comenzará a poner pequeños huevos y en pocas semanas llegará a poner huevos de tamaño medio deseando que ellas pongas huevos grandes.

Para la producción de huevos en reproductoras, se tienen en cuenta la edad, el peso corporal de las gallinas reproductoras y están relacionados con el tipo de alimentación.

Para determinar el efecto del tamaño y peso del huevo sobre la incubabilidad y rendimiento y características de la canal de pollos broilers se realizaron pocos trabajos, teniendo en cuenta que hay una estrecha relación positiva entre el tamaño del huevo y el tamaño del pollito nacido. Sin embargo, el tamaño del huevo puede ser manipulado por la alimentación, edad y peso de la gallina reproductora.

Asuquo et al. (1993) estudiaron los efectos de la edad en gallina reproductora y el tamaño del huevo sobre la fertilidad y la incubabilidad. Los autores observaron que el peso del huevo en un rango de 45 a 56 gramos tiene una mejor incubabilidad que los pequeños huevos. Además indican, que el tamaño del huevo afecta la incubabilidad más que el tamaño de los pollos. Porque el tamaño del huevo afecta principalmente en el retardo de la absorción del saco amniótico en los pollitos recién nacidos.

Abiola et al. (2008), en un trabajo sobre el efecto del huevo en la incubabilidad en pollos Anak, reporta que hay una estrecha relación positiva entre el tamaño del huevo y el tamaño del pollito nacido. Asi mismo, Senapati (1996) reportó una relación positiva entre el peso del huevo y el empollamiento en aves domésticas.

Tufft (1991), en un estudio sobre el efecto de la edad de la gallina con la retención de lípidos en el rendimiento del peso y sexo de los pollitos, encontró una alta correlación entre el peso del huevo y el peso del pollito.

Deeming, (1995), en un trabajo realizado con 24,000 huevos, reporta que el peso del huevo con 52 - $69 \mathrm{~g}$ presenta el peso del pollito con $64-67 \%$ del peso; los huevos grandes presentan mayor duración de la incubación y los huevos con cáscara fina presentan mayor pérdida de humedad, además no encajan bien en las bandejas, además indica que existe una alta correlación positiva con alta incubabilidad con la edad de las reproductoras. 
Asuquo et al. (1993) indican que las reproductoras al inicio de la puesta presentan menor proporción de yema, menor tamaño del huevo, mayor \% de huevos de dos yemas, mayor número de formas elípticas y mayor presencia de huevos no fecundados

Tufft (1991) indica que al final de la puesta los huevos presentan cáscaras de mala calidad, por el envejecimiento de las gallinas y de un envejecimiento más rápido de los machos.

Senapati (1996), con referencia al almacenamiento de los huevos incubables, este mecanismo prolonga el periodo de incubación, disminuye la tasa de eclosión, además puede deprimir el peso del pollito de un día cuando los huevos son almacenados por más de 15 días

Abiola et al. (2008) indican que al nacer los pollitos requieren de una humedad relativa alta porque favorece la rotura de la cascara, estimula los movimientos de los músculos respiratorios del pollito y requieren de una ventilación de 4,5 - 5,2 litros/hora y huevo.

El tamaño del huevo ha sido ampliamente estudiado en el contexto de la historia de la vida en el cual existen teorías ampliamente variables, algunos estudios reportaron que puede afectar tanto la salud de las reproductoras como la salud de los pollitos. El objetivo del presente trabajo fue determinar el efecto del tamaño y peso del huevo en su incubabilidad.

\section{MATERIALY MÉTODOS}

Se trabajaron con huevos procedentes de gallinas reproductoras de la línea Coob de la empresa Incubadora del Sur, distribuidas en 3 categorías:

Los huevos provinieron de gallinas reproductoras cuyas edades fueron de 22 a 32 semanas; los huevos fueron numerados y pesados individualmente utilizando una balanza de precisión, luego fueron categorizados como sigue:

Huevos pequeños: 41.09 - 50.97 gramos

Huevos medianos: 50.98 - 57.39 gramos

Huevos grandes: $57.40-69,64$ gramos

En el experimento se consideraron tres tratamientos con tres replicaciones por tratamiento, cada tratamiento con 66 huevos, con 22 huevos por replicación.

Los huevos fueron fumigados con formalina $y$ permanganato de potasio en relación de $1: 2$ por 15 minutos.

La temperatura óptima de la incubadora fue de $28.2^{\circ} \mathrm{C}$ con una humedad relativa de $83.5 \%$.

Los parámetros fueron monitoreados incluyendo pérdida de peso a la incubación, porcentaje de incubabilidad y porcentaje de mortalidad.

\section{Definiciones}

- Peso a la incubación: Peso de los huevos al inicio de la incubación

- Porcentaje de incubabilidad: Huevos fertilizados que entran en desarrollo embrionario

- Porcentaje de mortalidad: Muerte embrionaria a los 12 días de incubación.

En el análisis estadístico se utilizó un diseño completamente randomizado, los datos obtenidos fueron sujetos a un análisis de varianza de una vía usando el método de Snedecor and Cochran (1980). La prueba de Duncan fue utilizada para estudiar las diferencias entre medias como lo describe Gómez y Gómez (1986).

El modelo estadístico fue el siguiente:

$Y_{(i j)}=\mu+t i+\xi j(i)$

\section{Donde:}

$\mathrm{Y}=$ todas las observaciones (incubabilidad, mortalidad).

$\mu=$ promedio general de la población sobre la cual se está trabajando.

$\mathrm{t}=$ es la variación que se atribuye a los niveles del factor que se está evaluando (efecto de los tratamientos como: pequeño, mediano y grande).

$\xi=$ es la variación de los factores no controlados (error experimental).

$\mathrm{i}=\mathrm{i}$-ésimo tratamiento.

$\mathrm{j}=\mathrm{j}$-ésima repetición de cada tratamiento.

$\mathrm{j}(\mathrm{i})$ = es la variación de las unidades experimentales anidada en los tratamientos.

\section{RESULTADOS Y DISCUSIÓN}

Como se puede observar en el Cuadro $\mathrm{N}^{\circ} 01$, los valores obtenidos para la pérdida de peso de las tres categorías (grande, mediano y pequeño): se registró un valor mas alto en huevos medianos con relación a los otros; sin embargo, los huevos grandes registraron valores menores. No habiendo diferencias estadísticas significativas entre grupos. La similitud de estos resultados puede sugerir que las categorías con relación al tamaño de los huevos utilizados en el presente estudio probablemente tienen igual proporción de zonas de poro e igual diámetro de poro, independientemente del tamaño del huevo Renema (2001) indica que los huevos que presentan pérdidas de peso menores al $10 \%$ o encima del $20 \%$ de su masa inicial tienen menos probabilidades de eclosionar. El autor lo atribuye a la funcionalidad de la porosidad de la cáscara y la masa inicial de cada huevo.

Los resultados encontrados en el presente trabajo son similares a los reportados por Asuquo (1993) en un estudio sobre efectos de la edad de las reproductoras, con el tamaño del huevo indica pérdidas de peso entre 10 a $14 \%$ del peso del huevo a la primera semana de incubación. 
Tabla $\mathrm{N}^{\circ}$ 01: Pérdida de peso, porcentaje de incubabilidad y porcentaje de mortalidad según tamaño y peso del huevo

\begin{tabular}{|c|cc|c|c|c|}
\hline TAMAÑ & PESO & FÉRDIDADE PESO \% & \% INCURABIIIDAD & \% MORTALIDAD \\
\hline Grande & 57.40 & 69,64 & 10,7 & 82,88 & 12,42 \\
\hline Mediano & 50.98 & 57.39 & 12,6 & 96,65 & 2,56 \\
\hline Pequeño & 41.09 & 50.97 & 11,3 & 91,33 & 8,32 \\
\hline
\end{tabular}

Referente al porcentaje de incubabilidad se encontraron valores entre 82.88 a 96.65 , siendo el mayor encontrado en huevos de tamaño mediano y menor en huevos de tamaño grande. Estos resultados son similares a los encontrados por Tufft (1991), quien reporta una alta incubabilidad de huevos de tamaño mediano, sin embargo, Deeming (1995) indica que huevos pequeños constituyen una alta proporción de los huevos puestos por las pollitas y está fuertemente correlacionada con el peso del huevo.

Esta variabilidad de resultados en el presente estudio favoreciendo con alto porcentaje en huevos de tamaño mediano, puede deberse a que la masa del huevo mediano es adecuado genéticamente para el desarrollo del embrión.

En la mortalidad embrionaria se encontraron valores altos en huevos de tamaño grande con relación a los huevos medianos y pequeños, estos resultados pueden deberse a fallas de la incubadora como lo indica Buxadé (2000); sin embargo, Morris (1968) en un estudio sobre mortalidad embrionaria concluye que la fecundidad no mostró ninguna tendencia coherente con el peso del huevo para los huevos por encima de $46 \mathrm{~g}$., pero por debajo de los $46 \mathrm{~g}$. existe una tendencia de disminución de la fecundidad y en porcentajes de nacimientos

Tabla 2: Análisis de varianza para la mortalidad embrionaria

\begin{tabular}{|c|c|c|c|c|}
\hline $\begin{array}{l}\text { Fuente } \\
\text { variación }\end{array}$ & de GL. & $\begin{array}{l}\text { Suma } \\
\text { cuadrados }\end{array}$ & F & $\mathrm{Pr}<\mathrm{F}$ \\
\hline Tratamiento & 2 & 1,4418 & 6,42 & 0,0010 \\
\hline Lote & 1 & 0,0219 & 0,20 & 0,6587 \\
\hline Trat. Lote & 2 & 0,1536 & 0,68 & 0,5053 \\
\hline Error & 240 & 26,9291 & & \\
\hline Total & 245 & 28,5732 & & \\
\hline
\end{tabular}

Tabla 3: Mortalidad para los diferentes tratamientos

\begin{tabular}{|lll|}
\hline tratamiento & Medias & Error típico \\
\hline 1 Control & $9,62^{\mathrm{a}}$ & 0,037 \\
$2(0,02 \mathrm{mg} / \mathrm{kg})$. & $6,48^{\mathrm{a}}$ & 0,037 \\
$3(4 \mathrm{mg} / \mathrm{kg})$. & $24,07^{\mathrm{b}}$ & 0,037 \\
\hline
\end{tabular}

\section{CONCLUSIONES}

Los huevos de tamaño mediano obtuvieron mejores resultados con relación a huevos de tamaño pequeño $y$ grande, ello podría ser adecuado para tener en cuenta en incubadoras para obtener buenos porcentaje de nacimientos

\section{REFERENCIAS BIBLIOGRÁFICAS}

Abiola, S.S. 1999. Effects of turning frequency of hen's egg in electric table-type incubator on weight losses, hatchability and mortality. Nig. Agr. J., 30: 77-82.

ASUQUO, B.O. and B. OKON. 1993. Effects of age in lay and egg size on fertility an hatchability of chicken eggs. Nig. J. of Anim. Prod, 20: 122-124.

BUXADÉ C.C. 2000. La gallina ponedora. Ed. MuniPrensa, Madrid

DEEMING, D.C. 1995. Factors affecting hatchability during commercial incubation of ostrich (Struthio camelus) eggs. Brit. Poultry Sci., 36: 51-56.

GÓMEZ, K. A. and GÓMEZ A.A., 1986, Statistical Procedures for Agricultural Research. 2da Ed. Jhon Wiley and Sons Inc, USA

MORRIS, E.H., D.F. HESSELS, and R.J.BISHOP, 1968. The relationship between hatching egg weight and subsequent performance of broiler chickens. $\mathrm{Br}$. Poult. Sci. 9:305-315.

NORTH, M.O. and D.B. Bell, 1990. Commercial Chiken Production Manual. 4th ed. Van Nostrand Reinhold. New York, NY

RENEMA, R.A., 2001, Improvement of incubation egg quality by nutrition. II simpósio Internacional sobre nutriçäo de Aves. Concordia - SC-Brazil pp29-41

SENAPATI, P.K., K.G. DASK MADAL end A.K. CHATTERJJE. 1996, Relationship between egg weight, shape index, fertility and hatchability of Japanese quail eggs. Poultry Sci. 96: 1258 - 1262

TUFFT, L.S. and L.S. JENSEN. 1991. Effect of age of hen egg weight and sex on chick performance and lipid retention. Poultry Sci. 70:2411-2418 


\section{ANEXO}

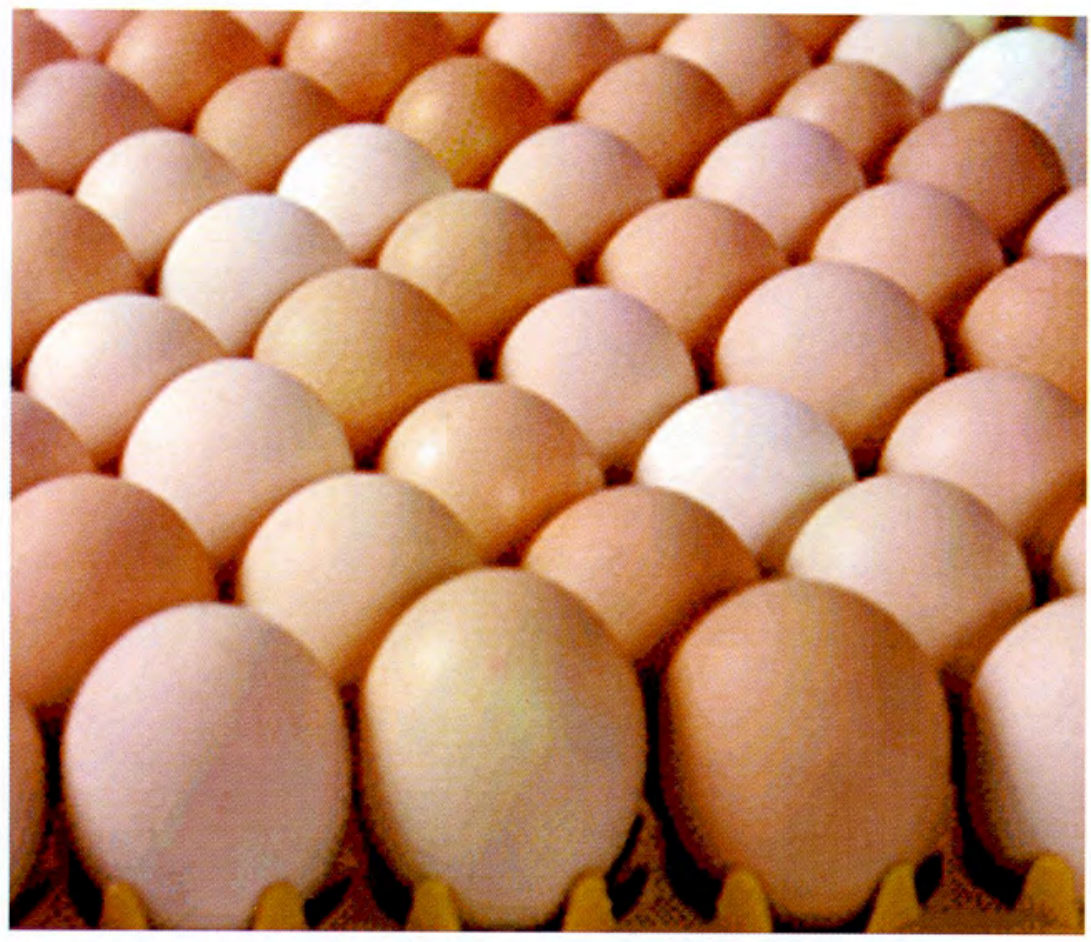

Fig. $\mathrm{N}^{\circ} 01$ Huevos seleccionados mostrando uniformidad de tamaño

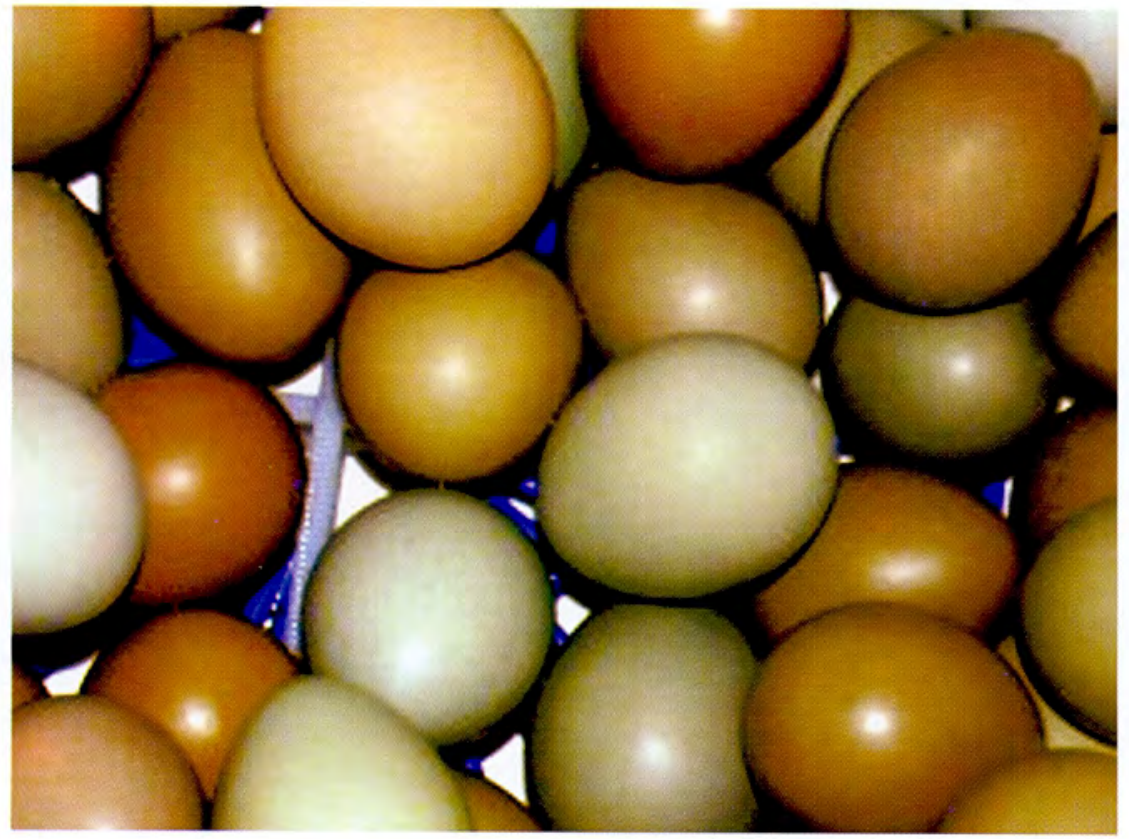

Fig. $\mathrm{N}^{\circ} 02$ Huevos de diferentes tamaños y tiempo de puesta 